\title{
OPEN Revealing noncollinear magnetic ordering at the atomic scale via XMCD
}

\author{
Fridtjof Kielgast ${ }^{1 凶}$, Ivan Baev ${ }^{1}$, Torben Beeck ${ }^{1}$, Federico Pressacco ${ }^{2}$ \& Michael Martins ${ }^{1}$
}

Mass-selected $\mathrm{V}$ and Fe monomers, as well as the heterodimer $\mathrm{Fe}_{1} \mathrm{~V}_{\mathbf{1}}$, were deposited on a $\mathrm{Cu}(001)$ surface. Their electronic and magnetic properties were investigated via X-ray absorption (XAS) and $X$-ray magnetic circular dichroism (XMCD) spectroscopy. Anisotropies in the magnetic moments of the deposited species could be examined by means of angle resolving XMCD, i.e. changing the $X$-ray angle of incidence. A weak adatom-substrate-coupling was found for both elements and, using group theoretical arguments, the ground state symmetries of the adatoms were determined. For the dimer, a switching from antiparallel to parallel orientation of the respective magnetic moments was observed. We show that this is due to the existence of a noncollinear spin-flop phase in the deposited dimers, which could be observed for the first time in such a small system. Making use of the two magnetic sublattices model, we were able to find the relative orientations for the dimer magnetic moments for different incidence angles.

Small magnetic adsorbate systems have been a focus of interest in recent years, as they are promising candidates for high-density data storage devices ${ }^{1,2}$. Beyond possible applications, the investigation of electronic and magnetic properties of adsorbates provides a way to study coupling phenomena on a fundamental level. It has been shown that the geometric constraints, imposed by a substrate, as well as the hybridization of the substrate with the adsorbate electronic states are often responsible for the appearance of large magnetic moments and anisotropies in magnetic adatoms $\mathrm{s}^{3}$. Especially in $3 d$ transition metals, a large number of interactions are present at comparable energy scales which leads to a complex behaviour and thus hinders theoretical predictions ${ }^{4}$. It is this complex interplay that is also at the basis of noncollinear magnetic systems.

A prominent example of such complex behaviour are $\mathrm{Fe}_{1-x} \mathrm{~V}_{x}$ bulk alloys, which are found to exist in a complex tetragonal structure called the $\sigma$-phase in a $\mathrm{V}$ content range from $\mathrm{x} \approx 30$ at $\%$ to 60 at $\%{ }^{5}$. It has been shown to behave as a re-entrant magnetic system which, upon lowering of the temperature, undergoes a transition from a paramagnetic to a ferromagnetic state and finally becomes a spin glass ${ }^{6}$. This calls for a closer look into the fundamental coupling between the two constituents. As a prototype model system, we prepared adsorbate systems with small amounts of the respective adatoms and the $\mathrm{Fe}_{1} \mathrm{~V}_{1}$ dimer on a $\mathrm{Cu}(001)$ surface. The choice of $\mathrm{Cu}(001)$ is motivated by its $s p$-like density of states at the Fermi edge and the low-lying $d$-bands (see e.g. ${ }^{7}$ ), which provide the possibility for a weak substrate-adsorbate hybridization. Results found for $\mathrm{Fe}$ adatoms on $\mathrm{Cu}(111)$, where multiplet features have been observed, reinforce this ${ }^{8}$.

The element specificity of X-ray transitions makes XAS a well-suited technique to investigate the electronic structure of compound systems like the present one. Additionally, using circular polarized light makes the absorption signal sensitive to the magnetic moment of the probed element by X-ray magnetic circular dichroism $(\mathrm{XMCD})^{9,10}$. We have chosen to investigate the $L_{2,3}$ edges, i.e. the $2 p_{j} \rightarrow 3 d$ absorption (with $j=\frac{1}{2}$, $\frac{3}{2}$ ) of $\mathrm{V}$ and Fe since their electronic and magnetic properties of are mainly determined by their $3 d$ levels.

\section{Results and discussion}

Fe and V adatoms. First, we will discuss the electronic properties of the Fe and V adatoms on $\mathrm{Cu}(001)$. The background corrected absorption spectra across their respective $L_{2,3}$-edges, as well as the resulting dichroism spectra are shown in Fig. 1c-f. In order to compare the spectral changes, these spectra have been normalized to their respective $L_{3}$ peak values. For both adatoms, the spectra show features at the lower energy flanks of the respective $L_{2,3}$-peaks ( $\sim 515.7 \mathrm{eV}$ and $522.2 \mathrm{eV}$ for $\mathrm{V}_{1}$ and $\sim 707.4 \mathrm{eV}$ and $721.1 \mathrm{eV}$ for $\left.\mathrm{Fe}_{1}\right)$. These multiplet features are the result of the interaction of the $2 p$ core hole and the $3 d$ electrons and are typically found in atomic

${ }^{1}$ Universität Hamburg, Institut für Experimentalphysik, Luruper Chaussee 149, 22761 Hamburg, Germany. 'Deutsches Elektronensynchrotron (DESY) Photon Science, Notkestraße 85, 22607 Hamburg, Germany. ${ }^{\boxplus}$ email: fridtjof.kielgast@desy.de 
a

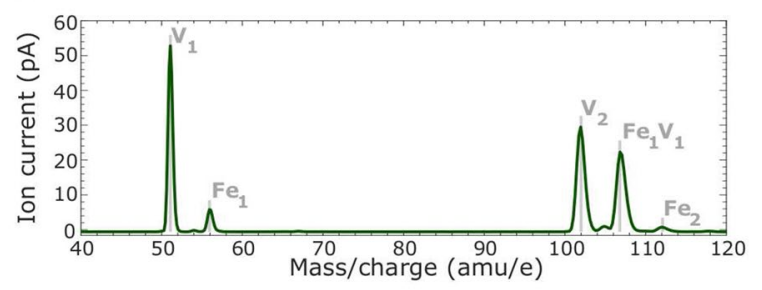

b
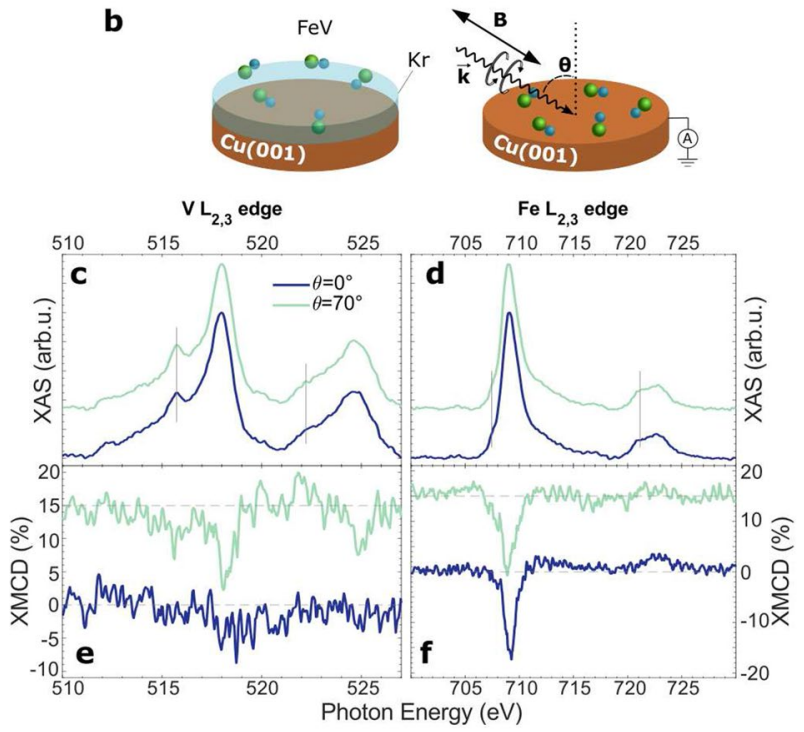

Figure 1. (a) Mass-to-charge spectrum of cluster ions up to $n=2$, produced from the FeV target. (b) Left: sketch of the soft landing procedure. Right: schematic view of the measurement geometry after the Kr buffer is removed by flash heating to $120 \mathrm{~K}$. The angle $\theta$ between the surface normal and the incoming photon beam is varied between $0^{\circ}$ and $70^{\circ}$. The magnetic field strength $B$ was alternated between -7 and $+7 \mathrm{~T}$. Background corrected white line spectra taken at $\theta=0^{\circ}$ and $\theta=70^{\circ}$ for $\mathrm{V}_{1} / \mathrm{Cu}(001)$ (c) and $\mathrm{Fe}_{1} / \mathrm{Cu}(001)$ (d). (e) and (f) are the XMCD spectra corresponding to $(\mathbf{c})$ and (d). All spectra are normalized to the $L_{3}$ peak value of the corresponding white line spectrum and vertically offset for better visibility.

\begin{tabular}{|l|l|l|}
\hline Adsorbate & Edges & BR \\
\hline $\mathrm{Fe}_{1}$ & $\mathrm{Fe} L_{2,3}$ & $0.83 \pm 0.04$ \\
\hline $\mathrm{V}_{1}$ & $\mathrm{~V} L_{2,3}$ & $0.66 \pm 0.03$ \\
\hline $\mathrm{Fe}_{1} \mathrm{~V}_{1}$ & $\mathrm{Fe} L_{2,3}$ & $0.79 \pm 0.03$ \\
\hline & $\mathrm{V} L_{2,3}$ & $0.58 \pm 0.03$ \\
\hline
\end{tabular}

Table 1. Branching ratios of the adsorbates for $\theta=0^{\circ}$ calculated from the white line spectra. Values for other incidence angles are omitted, as the branching ratio is found to be isotropic. For the alloy dimer, the branching ratio is shown for both elements separately.

and atomic-like species ${ }^{11-13}$. Comparison of Fig. 1c-d with XAS of free Fe and V atoms $\left(\mathrm{cf}^{11}{ }^{11}\right.$ ) shows very good agreement. The broadening and general energetic shift of the present spectra are due to the reduction of the Slater integrals, which is to be expected for systems showing some degree of hybridization ${ }^{14}$. The visibility of multiplet features and the resemblance of the spectra to those of free atoms implies a low degree of hybridization of the adatom $d$-states with the $\mathrm{Cu}(001)$ surface and ensures that the investigated magnetic properties are intrinsic to the adsorbates.

From the white line spectra, the so called branching ratio (BR) was obtained as the area under the $L_{3}$ edge divided by the area under the integrated $L_{3}$ and $L_{2}$ edges. Table 1 summarizes the results for the normal incidence spectra shown in Figs. 1c-d and $4 \mathrm{a}-\mathrm{b}$. As the branching ratios obtained in this work are independent of the angle of incidence, we restrict ourselves to the values for $\theta=0^{\circ}$. We want to point out, that the determination of the branching ratio for all $\mathrm{V}$ species suffers from some uncertainty due to the overlap of the $L_{3}$ and $L_{2}$ edges and the somewhat arbitrary separation of the two. However, in the context of this work, it is possible to compare the obtained branching ratios as the edges were separated in the same way consistently. 


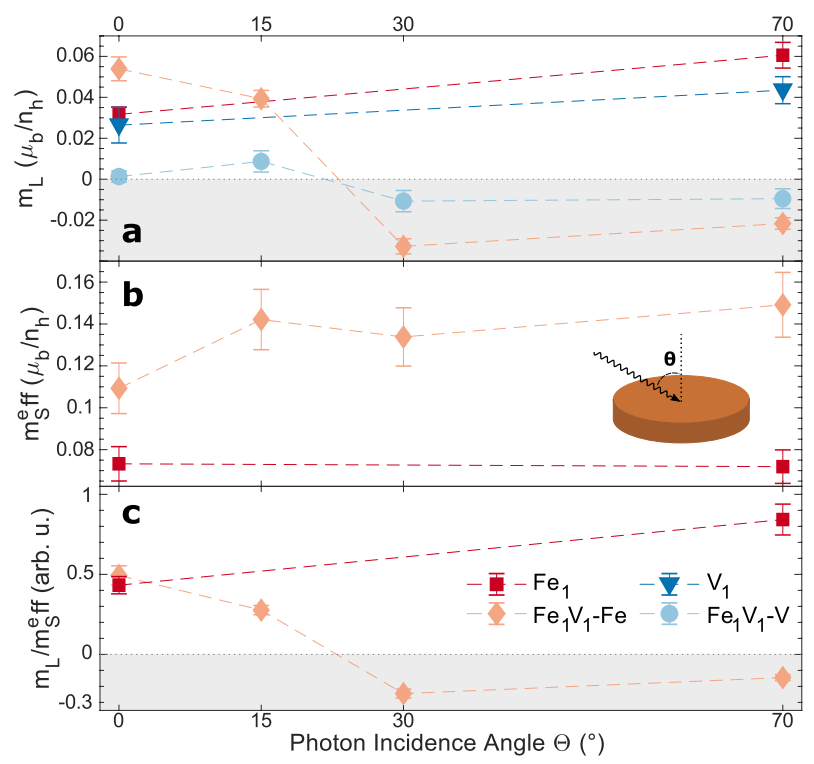

Figure 2. Orbital magnetic moments (a), effective spin magnetic moments (b) and ratio thereof (c) obtained via the sum rules ${ }^{20,21}$ for the investigated adsorbate systems as a function of photon incidence angle $\theta$. As the $\mathrm{V}$ $L_{2,3}$-edges are not separated well enough, only the orbital moments are calculated for those edges. The inset in (b) serves as a reminder of $\theta$ used in this work.

Considering only spin-orbit splitting in the initial $2 p$-states of Fe and V, one would expect the absorption intensity from $j=3 / 2$ to make up $2 / 3$ of the overall $2 p_{j} \rightarrow 3 d$ absorption intensity. This reflects the statistical degeneracy of the $2 p_{j}$-levels. However, the presence of spin-orbit coupling in the initial $3 d$-states as well as electrostatic interactions of the $2 p$ core-hole and the $3 d$ final states tend to change the measured intensity ratio ${ }^{15-17}$. The adsorbed monomers' branching ratios are, within the error bounds, identical to the ones obtained for free neutral atoms ${ }^{11}$ and cations ${ }^{12}$. They differ substantially from the branching ratios found for the respective bulk materials $(\mathrm{BR}(\mathrm{Fe})=0.70 \text { and } \mathrm{BR}(\mathrm{V})=0.50)^{16}$. It has previously been established that increased screening of the $2 p$ core, e.g. through hybridization with neighbouring atoms, lowers the branching ratio ${ }^{18,19}$. This is further evidence for a weak hybridization of the adatom and the substrate.

In the following, the magnetic properties of the $\mathrm{V}$ and Fe adatoms are discussed by means of the XMCD signals shown in Fig. 1(e-f). Using the well-established sum rules ${ }^{20,21}$, one can extract from the XMCD spectra the orbital magnetic moment $m_{L}$ and the effective spin magnetic moment $m_{S}^{e f f}=m_{S}+7 m_{T}$, where $m_{T}$ is the magnetic dipole moment, which accounts for non-spherical distributions of spin moments. The number of $3 d$-holes of the investigated atoms $\left(n_{h}\right)$, which appears as a factor in the equations for the sum rules, is not attainable through XMCD measurements alone. As we have neither a theoretical nor experimental value for this factor, we report the sum rule results per $n_{h}$. The small energetic separation of the $\mathrm{V} L$-edges would introduce a large error in the calculation of $\mathrm{m}_{S}^{e f f}$ and thus we only comment on the Fe spin moments. Although it is unlikely that the sample is in magnetic saturation, we can nevertheless refer to the values of the orbital and spin magnetic moments gained from the sum rules to compare measurements at different incidence angles, as they were all taken at the same magnetic field. Results of the sum rules applied to all spectra under investigation are plotted in Fig. 2.

For the $\mathrm{V}$ adatom, a weak but non-vanishing XMCD signal and hence a magnetic moment is found. This is in accordance with theoretical results ${ }^{22}$, where a magnetic moment of $3 \mu_{B}$ was found for $\mathrm{V} / \mathrm{Cu}(001)$, as well as experimental results for $\mathrm{V}$ embedded in $\mathrm{Cu}^{23}$. The $L_{3}$-XMCD-peak of the adsorbed $\mathrm{V}$ monomers XMCD is visibly stronger for $\theta=70^{\circ}$, indicating a preferred easy axis of magnetization which lies parallel to the $\mathrm{Cu}$ surface. This in-plane magnetization seen in the XMCD signal is confirmed by the anisotropy of the orbital moment (cf. Fig. 2a). For the Fe adatom, the height difference of the XMCD $L_{3}$-peaks is not so clear, and hence the orientation of the easy axis can not be determined as readily. However, the Fe orbital moment reveals a pronounced anisotropic behaviour favoring an in-plane orientation. This translates into an anisotropy in the $\mathrm{m}_{\mathrm{L}} / \mathrm{m}_{\mathrm{S}}^{\text {eff }}$ ratio (cf. Fig. 2c). With a value of $0.84 \pm 0.09$ for $\theta=70^{\circ}, \mathrm{m}_{\mathrm{L}} / \mathrm{m}_{\mathrm{S}}^{\text {eff }}$ is remarkably large and comparable to the one obtained for $\mathrm{Fe}$ on alkali metals, e.g. 0.95 for $\mathrm{Fe} / \mathrm{K}^{13}$, where the large orbital magnetic moment was attributed to a weak hybridization with the substrate.

Based on the measured in-plane anisotropy of both adatoms, we can gain further insight into the electronic ground state symmetry of the adsorbed monomers by using group theory. As both Fe and V are light transition metals with weak spin-orbit coupling ${ }^{24}$, they follow Russel-Saunders-coupling where the $l$ and $s$ quantum numbers of their $3 d$ electrons will, in a first step, couple to form $L$ and $S$ values. This results in ${ }^{5} D$ and ${ }^{4} F$ terms for the respective $d^{6}$ and $d^{3}$ configurations of Fe and V. The crystal field of the fourfold symmetric $\mathrm{Cu}(001)$ surface will then split these terms into irreducible representations of the $C_{4 v}$ point group. Geometric constraints only affect the spin through the spin-orbit coupling, thus one can focus primarily on the splitting of the orbital angular momentum. The ${ }^{5} D$ term of $d^{6}$ will split into four different irreducible terms, each of which transforms like the one-electron orbital indicated in brackets: ${ }^{5} \mathrm{~A}_{1}\left(d_{3 z^{2}-r^{2}}\right),{ }^{5} \mathrm{~B}_{1}\left(d_{x^{2}-y^{2}}\right),{ }^{5} \mathrm{~B}_{2}\left(d_{x y}\right)$ and the twofold degenerate ${ }^{5} \mathrm{E}$ 


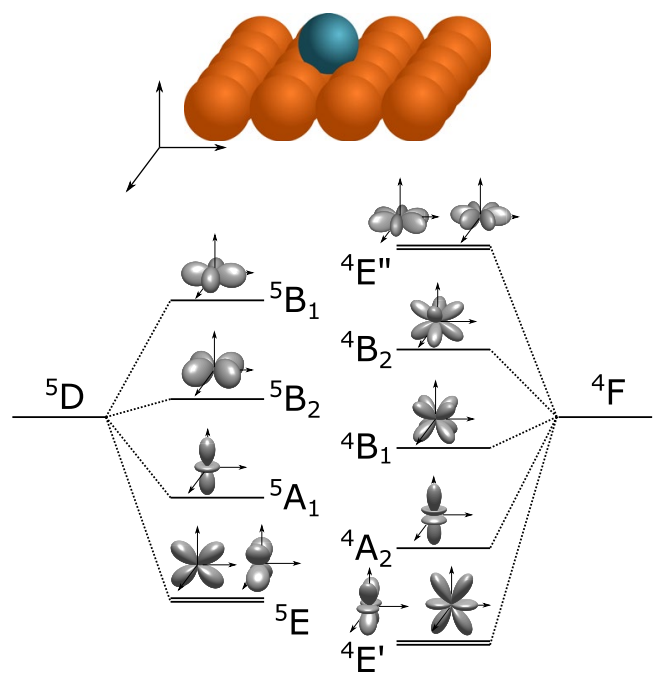

Figure 3. Sketch of the degeneracy lifting of the spherical symmetric ${ }^{5} D$ and ${ }^{4} F$ terms of the respective $d^{6}$ and $d^{3}$ configurations via the crystal field of a fourfold symmetric (001) surface and the form of the resulting irreducible representation. The new found representations are not ordered energetically.

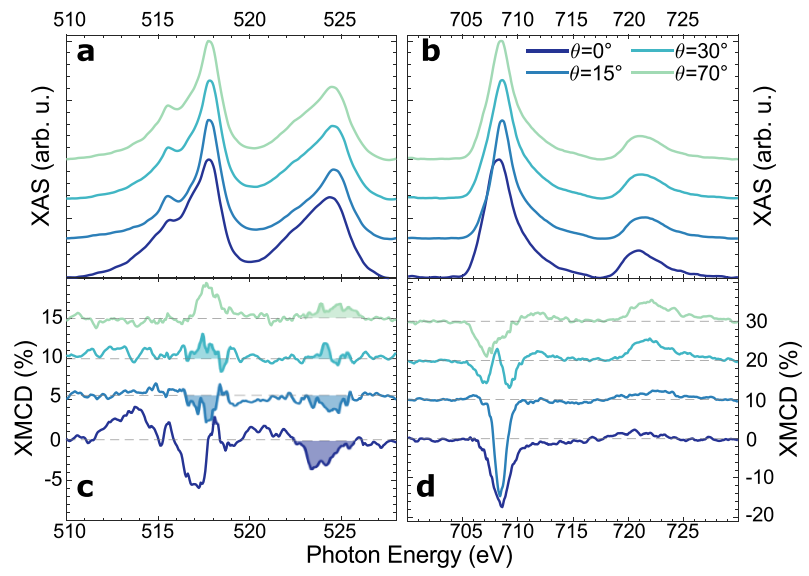

Figure 4. Background corrected white line spectra taken at $\theta=0^{\circ}, \theta=15^{\circ}, \theta=30^{\circ}$ and $\theta=70^{\circ}$ for the $\mathrm{V} L_{2,3}$-edges (a) and the Fe $L_{2,3}$-edges (b) of $\mathrm{Fe}_{1} \mathrm{~V}_{1} / \mathrm{Cu}(001)$. (c) and (d) are the XMCD spectra corresponding to (a) and (b). All spectra are normalized to the $L_{3}$ peak value of the corresponding white line spectrum and vertically offset for better visibility.

$\left(d_{x z}\right.$ and $\left.d_{y z}\right)$. The ${ }^{4} F$ term of $d^{3}$ will split into ${ }^{4} \mathrm{~A}_{2}\left(f_{z^{3}}\right),{ }^{4} \mathrm{~B}_{1}\left(f_{x y z}\right),{ }^{4} \mathrm{~B}_{2}\left(f_{z\left(x^{2}-y^{2}\right)}\right)$ and two $\mathrm{E}$ representations ${ }^{4} \mathrm{E}^{\prime}$ $\left(f_{y z^{2}}\right.$ and $\left.f_{x z^{2}}\right)$ and ${ }^{4} \mathrm{E}^{\prime \prime}\left(f_{y\left(3 x^{2}-y^{2}\right)} \text { and } f_{x\left(x^{2}-3 y^{2}\right)}\right)^{25}$. This splitting is schematically shown in Fig. 3 .

For $3 d$ metals, the crystal field splitting is considerably larger than the spin-orbit splitting ${ }^{4}$. Thus, the terms arising from the symmetry lowering will not be mixed or rearranged by spin-orbit coupling and we can identify the ${ }^{4} \mathrm{E}^{\prime}$ and ${ }^{5} \mathrm{E}$ terms as the respective ground states for the $\mathrm{Fe}$ and $\mathrm{V}$ adatom.

$\mathrm{Fe}_{1} \mathrm{~V}_{1} / \mathrm{Cu}(001)$. After having discussed the isolated $\mathrm{Fe}$ and $\mathrm{V}$ adatoms, we will bring both constituents together and study the $\mathrm{Fe}_{1} \mathrm{~V}_{1}$ dimer. The white line spectra of both elements are shown in Fig. $4 \mathrm{a}-\mathrm{b}$ for four different values of $\theta$, namely $0^{\circ}, 15^{\circ}, 30^{\circ}$ and $70^{\circ}$. This corresponds to an increased probing of in-plane molecular orbitals. Comparison of the normal incidence spectra with those of the adsorbed monomers shows a clear smearing of the multiplet features. We attribute this to an increased hybridzation and subsequent delocalization of the respective $d$-states in the dimer. This is consolidated by looking at Table 1, where a decrease in the branching ratio is observed for both species upon alloying. Due to the delocalization of the $d$-states, their overlap with the $2 p$ core hole diminishes, which reduces both the branching ratio and the strength of the multiplets.

Furthermore, the peak positions of the white line spectra shift to lower energies for both elements. For Fe, the $L_{3}$ peak shifts from $709.0 \mathrm{eV}$ to $708.4 \mathrm{eV}$, whereas the $\mathrm{V} L_{3}$ changes from $518.0 \mathrm{eV}$ to $517.8 \mathrm{eV}$ upon alloying. This shift is significant, given the energy resolution of $\approx 30 \mathrm{meV}$ of the P04 beamline at these photon energies ${ }^{26}$. Hirsch et al. have attributed such a lowering of the excitation energy to an increased dynamic screening of the $2 p$ 
core hole by the $3 d$ electrons in the final state ${ }^{12}$. The more efficient screening is a result of the stronger delocalization and subsequent increased mobility of the $3 d$ electrons. This reduces the energy of the final state, leading to a lowering of the absorption energy. In addition to the overall shift, there is a noticeable energetic shift for Fe when going from $\theta=0^{\circ}$ to $\theta=15^{\circ}$. We attribute this to a stronger contribution of an energetically lower orbital at normal incidence, leading to a shifting center of mass of the whole peak ${ }^{27,28}$.

Focusing now on the XMCD spectra of both elements shown in Fig. $4 \mathrm{c}-\mathrm{d}$, it can be seen that the normal incidence XMCD spectrum of V shows a positive onset at the $L_{3}$ peak. In combination with the negative sign of the Fe $L_{3} \mathrm{XMCD}$ in the same measurement geometry, this implies an antiferromagnetic coupling between the two constituents' magnetic moments. This is in accordance with results for Fe/V/Fe trilayer systems ${ }^{29}$ and $\mathrm{Fe}_{0.9} \mathrm{~V}_{0.1}$ alloys ${ }^{30}$, as well as electronic structure calculations of the free $\mathrm{FeV}$ dimer, where magnetic moments of $-2.8 \mu_{B}$ and $1.8 \mu_{B}$ were found for $\mathrm{V}$ and Fe respectively ${ }^{31}$. We want to note that the shape of the V XMCD is considerably different from the results of Wende et al. ${ }^{29}$ and Scherz et al. ${ }^{30}$. We explain this discrepancy by noting that the investigated system resembles an atomic species more than a bulk species and that subsequently the XMCD shows notably different features compared to the bulk ${ }^{32,33}$.

For $\theta=15^{\circ}$, the $\mathrm{V} \mathrm{XMCD} \mathrm{signal} \mathrm{decreases} \mathrm{strongly} \mathrm{in} \mathrm{magnitude} \mathrm{and} \mathrm{for} \mathrm{incidence} \mathrm{angles} \mathrm{larger} \mathrm{than} 15^{\circ}$, there is a visible sign flip in the V XMCD (cf. shaded areas in Fig. 4c). This indicates a change from antiparallel to parallel orientation of the $\mathrm{V}$ magnetic moment relative to the magnetic field. The absence of such an inversion in the Fe XMCD shows that the coupling of the $\mathrm{V}$ and Fe magnetic moments is more complex than a collinear (antiferromagnetic or ferromagnetic) configuration. From Fig. 2a it can be seen that the orbital moments of Fe and $\mathrm{V}$ in the dimer have the same sign for all incidence angles and we can thus identify the spin-spin interaction of $\mathrm{Fe}$ and $\mathrm{V}$ as giving rise to the observed noncollinear alignment. $\theta=30^{\circ}$ also marks the appearance of a splitting of the $L_{3}$ peak in the Fe XMCD into two structures, which are well separated for $\theta=30^{\circ}$ and can still be distinguished for $\theta=70^{\circ}$. Similar to the energy shift of the XAS at $\theta=0^{\circ}$ a possible explanation for this is the increased contribution of dimer orbitals of differing magnetic signature when increasing $\theta$. Another possibility would be the existence of different adsorption sites of the Fe atoms of the dimer, which in turn couple differently to the $\mathrm{V}$ atoms.

To explain the noncollinear configuration, we make use of the two magnetic sublattices model used, for example, $\mathrm{in}^{34}$. Assuming an uniaxial anisotropy, the free energy of a dimer can be expressed by Eq. (1). Here, the first term is the Zeeman energy, the second term is the exchange energy between Fe and $\mathrm{V}$ and the third and fourth terms represent the anisotropy energies of the constituents up to second order.

$$
E=-B\left(\mathrm{~m}_{\mathrm{Fe}} \cos \left(\alpha_{\mathrm{Fe}}-\theta\right)+\mathrm{m}_{\mathrm{V}} \cos \left(\alpha_{\mathrm{V}}-\theta\right)\right)-\mathrm{J}_{\mathrm{Fe}-\mathrm{V}} \mathrm{m}_{\mathrm{Fe}} \mathrm{m}_{\mathrm{V}} \cos \left(\alpha_{\mathrm{Fe}}-\alpha_{\mathrm{V}}\right)+\mathrm{K}_{\mathrm{uni}}^{\mathrm{Fe}} \sin ^{2}\left(\alpha_{\mathrm{Fe}}\right)+\mathrm{K}_{\mathrm{uni}}^{\mathrm{V}} \sin ^{2}\left(\alpha_{\mathrm{V}}\right)
$$

$\alpha_{\mathrm{Fe}, \mathrm{V}}$ denote the orientation angles of the magnetic moments $\mathrm{m}_{\mathrm{Fe}}$ and $\mathrm{m}_{\mathrm{V}}$ relative to the surface normal (cf. Fig. 5). $J_{\mathrm{Fe}-\mathrm{V}}$ is the exchange constant between the two materials and $K_{u n i}^{\mathrm{Fe}, \mathrm{V}}$ are their respective anisotropy constants. A stable configuration is found by numerically minimizing Eq. (1) with respect to $\alpha_{\mathrm{Fe}}$ and $\alpha_{\mathrm{V}}$. Depending on the interplay between exchange coupling and anisotropy, there exists a so-called spin-flop phase in which the magnetic field, applied along the easy axis of the system, causes the magnetic moments to cant away from the collinear case.

In the absence of reported magnetic moments for the present system, $\mathrm{m}_{\mathrm{Fe}}$ and $\mathrm{m}_{\mathrm{V}}$ were assumed to be $2.27 \mu_{B}$ and $0.99 \mu_{B}$, based on the $\mathrm{Fe}_{0.9} \mathrm{~V}_{0.1}$ alloy reported $\mathrm{in}^{30}$. Equation (1) was subsequently minimized for large ranges

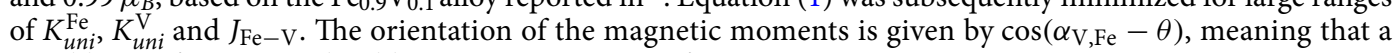
suitable set of constants should give $\cos \left(\alpha_{\mathrm{V}}-\theta\right)>0$ for $\theta \geq 30^{\circ}$.

An exemplary set of angles which were calculated to recreate the measurements are shown in Fig. 5(b). In this particular case, $J_{\mathrm{Fe}-\mathrm{V}}$ was $-0.575 \pm 0.005 \mathrm{meV} / \mu_{B}^{2}, K_{u n i}^{\mathrm{Fe}}$ was $1.80 \pm 0.01 \mathrm{meV} /$ atom and $K_{u n i}^{\mathrm{V}}$ was $-0.97 \pm 0.01 \mathrm{meV} /$ atom. These values were chosen as they also yield similar magnitudes for the V XMCD in the case of $\theta=15^{\circ}$ and $\theta=30^{\circ}$. While $K_{u n i}^{\mathrm{Fe}}$ is significantly higher than the corresponding value of bcc Fe $\left(K_{1} \approx 4 \mu \mathrm{eV} / \mathrm{atom}^{35}\right)$, it is still reasonable considering the reduced symmetry of the Fe atom within the dimer. Also, $K_{u n i}^{\mathrm{Fe}}$ is remarkably similar to the value of $K$ of $1.8 \mathrm{meV} /$ atom found for Fe monomers on $\mathrm{Cu}(111)^{8}$. Isberg et al. found a combined $K_{u n i}$ value of $0.3 \mathrm{meV}$ for $\mathrm{Fe} / \mathrm{V}(100)$ superlattices ${ }^{36}$. Although it is difficult to compare our results to this overall value, we want to note that the sum of $K_{u n i}^{\mathrm{Fe}}$ and $K_{u n i}^{\mathrm{V}}$ (namely $0.78 \mathrm{meV}$ ) turns out to be less than a factor of 3 off. Multiplying the best fit value of $J_{\mathrm{Fe}-\mathrm{V}}$ with the assumed magnetic moments of $\mathrm{Fe}$ and $\mathrm{V}$, we arrive at an exchange energy of $-1.035 \mathrm{meV}$, which is in reasonably good agreement with the antiferromagnetic exchange energy of $-0.94 \mathrm{meV}$ Poulopoulos et al. found for $\mathrm{Fe}_{n} \mathrm{~V}_{m}$ superlattices on $\mathrm{MgO}(001)^{37}$.

\section{Conclusions}

To summarize, we have studied the magnetic properties of $\mathrm{Fe}$ and $\mathrm{V}$ adatoms as well as the $\mathrm{Fe}_{1} \mathrm{~V}_{1}$ dimer on a $\mathrm{Cu}(001)$ surface using X-ray spectroscopic methods. The Fe and V adatoms interact weakly with the $\mathrm{Cu}(001)$ surface, leading to the appearance of multiplet features in the $L_{2,3}$-edge NEXAFS spectra. From spectra at two different incidence angles, an in-plane easy axis has been seen for both adatoms. The respective orientations of the individual magnetic moments in the dimer have been determined by taking XMCD spectra at different photon incidence angles, i.e. angular resolved XMCD at the corresponding $L_{2,3}$-edges. These results give evidence for the existence of a spin-flop phase already in the magnetic moments in a $\mathrm{Fe}_{1} \mathrm{~V}_{1}$ dimer on a $\mathrm{Cu}(001)$ surface. Employing a simple magnetic model, we were able to determine a set of constants that reproduce the measured behaviour. The magnitudes of these constants are consistent with the weak substrate-adsorbate interaction we found for the respective adatoms. The fundamental dimer system studied in this work already shows a complex noncollinear magnetic coupling and lends itself to the comparison to theoretical models, since many-body effects are less crucial in such a model system. 


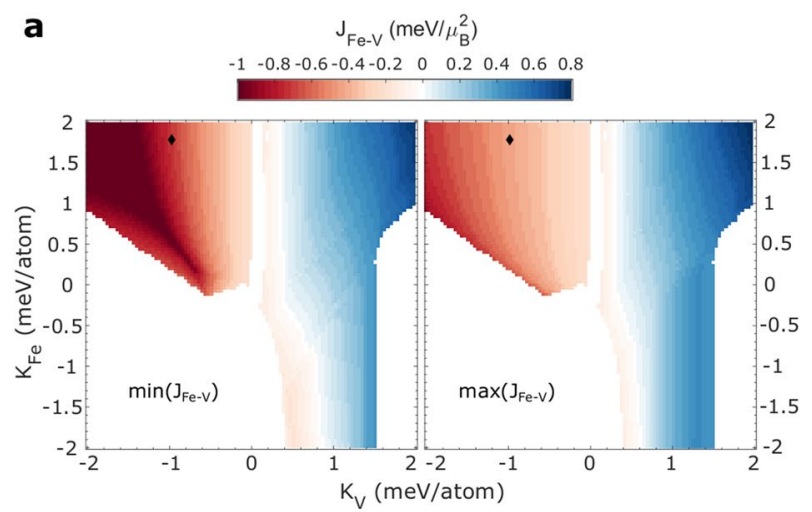

b

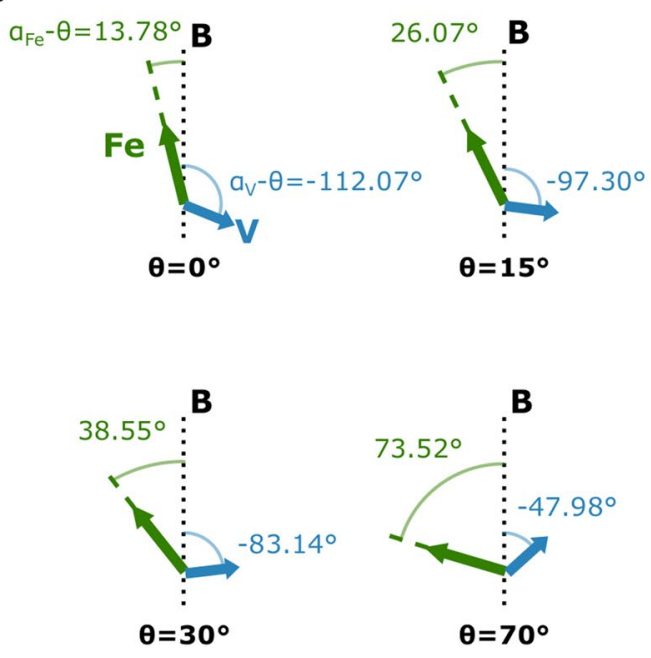

Figure 5. (a) Combinations of $J_{\mathrm{Fe}-\mathrm{V}}, K_{u n i}^{\mathrm{Fe}}$ and $K_{u n i}^{\mathrm{V}}$ which allow a spin-flop phase at a magnetic field strength of $7 \mathrm{~T}$ and magnetic moments of $2.27 \mu_{B}$ and $0.99 \mu_{B}$ for Fe and V respectively. For every pair of $K_{u n i}^{\mathrm{V}}$ and $K_{u n i}^{\mathrm{Fe}}$, all nonzero values of $J_{\mathrm{Fe}-\mathrm{V}}$ between the lower bound $\min \left(J_{\mathrm{Fe}-\mathrm{V}}\right)$ (left) and the upper bound $\max \left(J_{\mathrm{Fe}-\mathrm{V}}\right)($ right $)$ produce a noncollinear arrangement. The black diamond indicates the best fit to the observed XMCD height ratios. These are $J_{\mathrm{Fe}-\mathrm{V}}=-0.575 \pm 0.005 \mathrm{meV} / \mu_{B}^{2}, K_{u n i}^{\mathrm{Fe}}=1.80 \pm 0.01 \mathrm{meV} /$ atom and $K_{u n i}^{\mathrm{V}}=-0.97 \pm 0.01 \mathrm{meV} /$ atom. (b) Lowest energy orientations of the magnetic moments relative to the magnetic field, corresponding to the values indicated by the diamond in (a) for the four incidence angles used in this work.

Going forward, it would be interesting to compare the present results with XMCD measurements on the free $\mathrm{Fe}_{1} \mathrm{~V}_{1}$ dimer and to perform similar measurements on a wide range of dimer constituents and surfaces to establish systematic information on magnetic coupling at these scales.

\section{Methods}

The preparation and deposition of monomers and dimers, as well as the X-ray absorption measurements were performed in situ and under UHV conditions at the P04 beamline ${ }^{26}$ at PETRA III in Hamburg. The low base pressure $\left(\leq 5 \times 10^{-10} \mathrm{mbar}\right)$ during preparation and measurement is crucial in assuring that no oxidation of the adsorbates occurs, which would critically alter their electronic structure and magnetic properties. For the same reasons, and to ensure a reproducible substrate, the $\mathrm{Cu}$ surface was sputter cleaned and annealed extensively prior to each preparation.

Following ${ }^{38}$, adatoms and clusters were produced by sputtering a $\mathrm{FeV}$ target ( $50 / 50$ at $\%$ with purity $>99.9 \%$ ) with $30 \mathrm{keV} \mathrm{Xe}{ }^{+}$ions. The cationic sputter fragments were extracted from the sputter region via a potential of $500 \mathrm{~V}$. Mass selection of the fragments due to their mass-to-charge ratio was achieved in a dipole magnetic field perpendicular to the fragments trajectory. The resulting mass spectrum is shown in Fig. 1a. The mass-selected fragments are landed with a kinetic energy of $\sim 1 \mathrm{eV} /$ atom and a soft landing scheme ${ }^{39,40}$ is employed to avoid fragmentation or implantation of the adsorbates into the substrate. Kr buffer layers were adsorbed onto the substrate at $\mathrm{T} \approx 25 \mathrm{~K}$ before cluster deposition and desorbed afterward by flash heating to $\mathrm{T} \approx 120 \mathrm{~K}$. Figure $1 \mathrm{~b}$ shows a schematic of the deposition process. The low substrate temperature of $\sim 25 \mathrm{~K}$ in combination with the low coverage of adsorbates on the surface $(<0.03 \mathrm{ML})$, serve to prevent adsorbate diffusion and agglomeration.

The $\mathrm{V} L_{2,3}$ and $\mathrm{Fe} L_{2,3}$-edge spectra of the deposited species were recorded by scanning the photon energy in the ranges of $500-528 \mathrm{eV}$ and $700-730 \mathrm{eV}$, respectively. For the present case, the energy resolution of the beamline 
was $\sim 30 \mathrm{meV}^{26}$. The sample drain current, representing the total electron yield (TEY), was used as a measure of the X-ray absorption. To minimize the influence of possible fluctuations of the photon flux on the absorption, measurements were normalized to the TEY signal of an Au grid placed half a meter before the sample.

The XMCD signal was obtained as the difference of absorption spectra for parallel $\left(\mu_{+}\right)$and antiparallel $\left(\mu_{-}\right)$ alignment of the photon wavevector $\mathbf{k}$ and the magnetic field $\mathbf{B}$. In contrast to our earlier work on mass-selected adsorbates ${ }^{41-44}$, alignment of the magnetic moments was achieved via a superconducting split pair solenoid magnet with maximum magnetic field of $\pm 7 \mathrm{~T}$, purchased from Cryogenic Limited ${ }^{45}$. The polarization of the photon beam was kept fixed and collinear to the magnetic field, which was switched between the two extrema. Angular dependence of the adsorbate magnetic moments was investigated by rotating the sample, while keeping the respective directions of the photon wavevector $\mathbf{k}$ and the magnetic field $\mathbf{B}$ fixed, and hence changing the photon angle of incidence relative to the surface normal ( $\theta$ in Fig. 1b).

Given the dilute nature of the adsorbates, the absorption spectrum is dominated by the Cu substrate. As the investigated energy range lies far away from an X-ray absorption edge of the substrate, its contribution is treated as a linear background and subtracted from the spectra. Furthermore, a double step function was subtracted from all the spectra accounting for the absorption signal from Fe and V electrons being excited into $s p$-like states.

Received: 31 August 2020; Accepted: 18 January 2021

Published online: 04 February 2021

\section{References}

1. Lau, J. W. \& Shaw, J. M. Magnetic nanostructures for advanced technologies: fabrication, metrology and challenges. J. Phys. D: Appl. Phys. 44, 303001. https://doi.org/10.1088/0022-3727/44/30/303001 (2011).

2. Loth, S., Baumann, S., Lutz, C. P., Eigler, D. M. \& Heinrich, A. J. Bistability in atomic-scale antiferromagnets. Science 335, 196-199. https://doi.org/10.1126/science.1214131 (2012).

3. Brune, H. \& Gambardella, P. Magnetism of individual atoms adsorbed on surfaces. Surf. Sci. 603, 1812-1830. https://doi. org/10.1016/j.susc.2008.11.055 (2009).

4. Stöhr, J. X-ray magnetic circular dichroism spectroscopy of transition metal thin films. J. Electron Spec. Relat. Phenom. 75, $253-272$. https://doi.org/10.1016/0368-2048(95)02537-5 (1995).

5. Dubiel, S. M. \& Cieślak, J. Sigma-phase in Fe-Cr and Fe-V alloy systems and its physical properties. Crit. Rev. Solid State Mater. Sci. 36, 191-208. https://doi.org/10.1080/10408436.2011.589232 (2011).

6. Barco, R., Pureur, P., Fraga., G. L. F. \& Dubiel, S. M. Extended scaling in the magnetic critical phenomenology of the $\sigma$-phase Fe0.53-Cr0.47 and Fe0.52-V0.48 alloys. J. Phys.: Condens. Matter 24, 046002. https://doi.org/10.1088/0953-8984/24/4/046002 (2012).

7. Smith, J. R., Gay, J. G. \& Arlinghaus, F. J. Self-consistent local-orbital method for calculating surface electronic structure: application to Cu (100). Phys. Rev. B 21, 2201-2221. https://doi.org/10.1103/PhysRevB.21.2201 (1980).

8. Pacchioni, G. E. et al. Multiplet features and magnetic properties of Fe on $\mathrm{Cu}(111)$ : From single atoms to small clusters. Phys. Rev. B 91, 235426. https://doi.org/10.1103/PhysRevB.91.235426 (2015).

9. Schütz, G. et al. Absorption of circularly polarized X rays in iron. Phys. Rev. Lett. 58, 737-740. https://doi.org/10.1103/PhysRevLet t.58.737 (1987).

10. Chen, C. T., Sette, F., Ma, Y. \& Modesti, S. Soft-X-ray magnetic circular dichroism at the $l_{2,3}$ edges of nickel. Phys. Rev. B 42, 7262-7265. https://doi.org/10.1103/PhysRevB.42.7262 (1990).

11. Martins, M., Godehusen, K., Richter, T., Wernet, P. \& Zimmermann, P. Open shells and multi-electron interactions: core level photoionization of the 3d metal atoms. J. Phys. B 39, R79-R125. https://doi.org/10.1088/0953-4075/39/5/r01 (2006).

12. Hirsch, K. et al. Initial- and final-state effects on screening and branching ratio in $2 p \mathrm{x}$-ray absorption of size-selected free $3 d$ transition metal clusters. Phys. Rev. B 86, 165402. https://doi.org/10.1103/PhysRevB.86.165402 (2012).

13. Gambardella, P. et al. Localized magnetic states of Fe Co, and Ni impurities on alkali metal films. Phys. Rev. Lett. 88, 047202. https ://doi.org/10.1103/PhysRevLett.88.047202 (2002).

14. Lynch, D. W. \& Cowan, R. D. Effect of hybridization on $4 \mathrm{~d} \rightarrow 4 \mathrm{f}$ spectra in light lanthanides. Phys. Rev. B 36, 9228-9233. https:// doi.org/10.1103/PhysRevB.36.9228 (1987).

15. Thole, B. T. \& van der Laan, G. Branching ratio in X-ray absorption spectroscopy. Phys. Rev. B 38, 3158-3171. https://doi. org/10.1103/PhysRevB.38.3158 (1988).

16. Fink, J. et al. 2p absorption spectra of the 3d elements. Phys. Rev. B 32, 4899-4904. https://doi.org/10.1103/PhysRevB.32.4899 (1985).

17. Zaanen, J., Sawatzky, G. A., Fink, J., Speier, W. \& Fuggle, J. C. $L_{2,3}$ absorption spectra of the lighter 3 d transition metals. Phys. Rev. B 32, 4905-4913. https://doi.org/10.1103/PhysRevB.32.4905 (1985).

18. Schwitalla, J. \& Ebert, H. Electron core-hole interaction in the X-ray absorption spectroscopy of $3 d$ transition metals. Phys. Rev. Lett. 80, 4586-4589. https://doi.org/10.1103/PhysRevLett.80.4586 (1998).

19. Ankudinov, A. L., , A. I. N. \& , J. J. R. Dynamic screening effects in X-ray absorption spectra. Phys. Rev. B 67, 115120. https://doi. org/10.1103/PhysRevB.67.115120 (2003)

20. Carra, P., Thole, B., Altarelli, M. \& Wang, X. X-ray circular dichroism and local magnetic fields. Phys. Rev. Lett. 70, 694-697. https ://doi.org/10.1103/PhysRevLett.70.694 (1993).

21. Thole, B. T., Carra, P., Sette, F. \& van der Laan, G. X-ray circular dichroism as a probe of orbital magnetization. Phys. Rev. Lett. 68, 1943-1946. https://doi.org/10.1103/PhysRevLett.68.1943 (1992).

22. Stepanyuk, V. et al. Metamagnetic states of $3 \mathrm{~d}$ nanostructures on the $\mathrm{Cu}(001)$ surface. J. Magn. Magn. Mater. 165, 272-274. https ://doi.org/10.1016/S0304-8853(96)00527-6 (1997).

23. Huttel, Y., van der Laan, G., Teodorescu, C. M., Bencok, P. \& Dhesi, S. S. Magnetic V embedded in copper evidenced by x-ray magnetic circular dichroism. Phys. Rev. B 67, 052408. https://doi.org/10.1103/PhysRevB.67.052408 (2003).

24. van der Laan, G. Microscopic origin of magnetocrystalline anisotropy in transition metal thin films. J. Phys.: Condens. Matter 10, 3239-3253. https://doi.org/10.1088/0953-8984/10/14/012 (1998).

25. Dresselhaus, M. S., Dresselhaus, G. \& Jorio, A. Group Theory: Application to the Physics of Condensed Matter (Springer, Berlin, Heidelberg, 2007).

26. Viefhaus, J. et al. The variable polarization XUV beamline P04 at PETRA III: Optics, mechanics and their performance. Nucl. Instrum. Methods Phys. Res. Sect. A 710, 151 - 154. https://doi.org/10.1016/j.nima.2012.10.110 (2013).

27. Stöhr, J., Baberschke, K., Jaeger, R., Treichler, R. \& Brennan, S. Orientation of chemisorbed molecules from surface-absorption fine-structure measurements: $\mathrm{CO}$ and $\mathrm{NO}$ on Ni(100). Phys. Rev. Lett. 47, 381-384. https://doi.org/10.1103/PhysRevLett.47.381 (1981). 
28. Rosenberg, R. A., Love, P. J. \& Rehn, V. Polarization-dependent C(K) near-edge X-ray-absorption fine structure of graphite. Phys. Rev. B 33, 4034-4037. https://doi.org/10.1103/PhysRevB.33.4034 (1986).

29. Wende, H. et al. XMCD analysis beyond standard procedures. AIP Conf. Proc. 882, 78-82. https://doi.org/10.1063/1.2644435 (2007).

30. Scherz, A. et al. Relation between $L_{2,3} \mathrm{XMCD}$ and the magnetic ground-state properties for the early $3 d$ element V. Phys. Rev. B 66, 184401. https://doi.org/10.1103/PhysRevB.66.184401 (2002).

31. Gutsev, G. L., Mochena, M. D., Jena, P., Bauschlicher, C. W. \& Partridge, H. Periodic table of 3d-metal dimers and their ions. J. Chem. Phys. 121, 6785-6797. https://doi.org/10.1063/1.1788656 (2004).

32. Zamudio-Bayer, V. et al. Large orbital magnetic moments of small, free cobalt cluster ions $\mathrm{Co}_{n}^{+}$with $\mathrm{n} \leqslant 9$. J. Phys.: Condens. Matter 30, 464002. https://doi.org/10.1088/1361-648x/aae54a (2018).

33. Zamudio-Bayer, V. et al. Direct observation of high-spin states in manganese dimer and trimer cations by X-ray magnetic circular dichroism spectroscopy in an ion trap. J. Chem. Phys. 142, 234301. https://doi.org/10.1063/1.4922487 (2015).

34. Radwański, R. J. Anisotropic ferrimagnets in high magnetic fields. Physica B+C 142, 57 - 64. https://doi.org/10.1016/03784363(86)90225-1 (1986).

35. Blundell, S. Magnetism in Condensed Matter. Oxford Master Series in Condensed Matter Physics (Oxford University Press, 2001).

36. Isberg, P. et al. Structure and magnetic properties of Fe/V (110) superlattices. Phys. Rev. B 57, 3531-3538. https://doi.org/10.1103/ PhysRevB.57.3531 (1998).

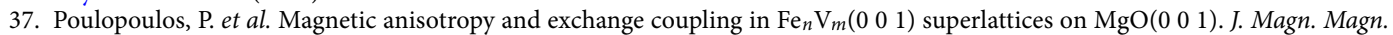
Mater. 170, 57-66. https://doi.org/10.1016/S0304-8853(97)00016-4 (1997).

38. Lau, J. T. et al. Ultrahigh vacuum cluster deposition source for spectroscopy with synchrotron radiation. Rev. Sci. Instrum. 76, 063902. https://doi.org/10.1063/1.1921551 (2005).

39. Lau, J. T., Wurth, W., Ehrke, H.-U. \& Achleitner, A. Soft landing of size-selected clusters in rare gas matrices. Low Temp. Phys. 29, 223-227. https://doi.org/10.1063/1.1542443 (2003).

40. Fedrigo, S., Harbich, W. \& Buttet, J. Soft landing and fragmentation of small clusters deposited in noble-gas films. Phys. Rev. B 58, 7428-7433 (1998).

41. Chen, K. et al. Oxidation-induced spin reorientation in Co adatoms and CoPd dimers on Ni/Cu(100). Phys. Rev. B 93, 144421. https://doi.org/10.1103/PhysRevB.93.144421 (2016).

42. Chen, K. et al. Hybridization and magnetism in small FePt alloy clusters. New J. Phys. 14, 123005. https://doi.org/10.1088/13672630/14/12/123005 (2012).

43. Glaser, L. et al. Magnetic properties of small size-selected Co and CoPt clusters on Ni. Phys. Rev. B 86, 075435. https://doi. org/10.1103/PhysRevB.86.075435 (2012).

44. Lau, J. T., Föhlisch, A., Nietubyc̀, R., Reif, M. \& Wurth, W. Size-dependent magnetism of deposited small iron clusters studied by x-ray magnetic circular dichroism. Phys. Rev. Lett. 89, 057201. https://doi.org/10.1103/PhysRevLett.89.057201 (2002).

45. Beeck, T. Small, Size-Selected and Deposited Clusters on Magnetic and Non-Magnetic 3d Transition Metal Surfaces. Ph.D. thesis, University of Hamburg (2015).

\section{Acknowledgements}

We acknowledge DESY (Hamburg, Germany), a member of the Helmholtz Association HGF, for the provision of experimental facilities. Parts of this research were carried out at PETRA III and we would like to thank Jens Viefhaus, Frank Scholz and Jörn Seltmann for assistance in using the P04 beamline. The authors gratefully acknowledge funding by the Deutsche Forschungsgemeinschaft under the SFB668 and by the German Federal Ministry of Education and Research (BMBF) within the contracts 05K16GUC and 05K19GU3. F. Pressacco acknowledges funding from the excellence cluster "The Hamburg Centre for Ultrafast Imaging - Structure, Dynamics and Control of Matter at the Atomic Scale" of the Deutsche Forschungsgemeinschaft (DFG Grant No. EXC 1074). In honor of Prof. Wilfried Wurth, who sadly passed away in the time between the beginning and the publication of this work.

\section{Author contributions}

F.K., I.B., T.B. and M.M. conducted the experiments. F.K. and F.P. analysed the results. All authors reviewed the manuscript.

\section{Funding}

Open Access funding enabled and organized by Projekt DEAL.

\section{Competing interests}

The authors declare no competing interests.

\section{Additional information}

Correspondence and requests for materials should be addressed to F.K.

Reprints and permissions information is available at www.nature.com/reprints.

Publisher's note Springer Nature remains neutral with regard to jurisdictional claims in published maps and institutional affiliations.

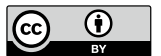

Open Access This article is licensed under a Creative Commons Attribution 4.0 International License, which permits use, sharing, adaptation, distribution and reproduction in any medium or format, as long as you give appropriate credit to the original author(s) and the source, provide a link to the Creative Commons licence, and indicate if changes were made. The images or other third party material in this article are included in the article's Creative Commons licence, unless indicated otherwise in a credit line to the material. If material is not included in the article's Creative Commons licence and your intended use is not permitted by statutory regulation or exceeds the permitted use, you will need to obtain permission directly from the copyright holder. To view a copy of this licence, visit http://creativecommons.org/licenses/by/4.0/.

(c) The Author(s) 2021 